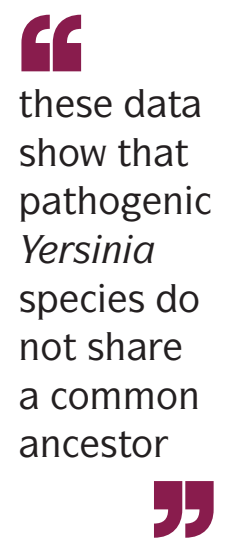

$\Rightarrow$ BACTERIAL EVOLUTION

\title{
Parting of the ways for Yersinia
}

The genus Yersinia mainly comprises environmental, non-disease causing species, but also includes human pathogens, such as Yersinia pestis, which is the causative agent of plague, and the enteropathogens Yersinia enterocolitica and Yersinia pseudotuberculosis. These pathogenic strains have been suggested to share a common ancestor; however, a new large-scale genomic analysis now shows that they evolved independently but have followed parallel evolutionary pathways.

By using whole-genome sequencing of 241 Yersinia strains, Reuter et al. defined a new phylogenetic tree for the Yersinia genus, based on a set of 84 core genes. This analysis confirmed previous observations that $Y$. pestis and Y. pseudotuberculosis are close relatives, but revealed a diametric separation

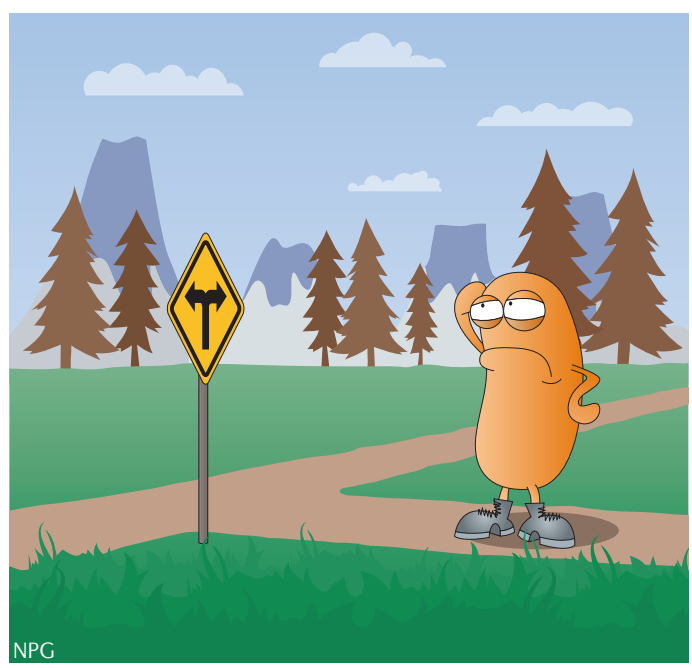

from $Y$. enterocolitica, with most environmental species occupying intermediate branching positions between these two clusters.

By analysing the distribution of known pathogenicity determinants across the Yersinia genus, the authors showed that multiple virulence pathways were selectively present within entire lineages. This includes the ability to degrade 1,2-propanediol or ethanolamine using vitamin B12-dependent enzymes - a pathway that is absent in the $Y$. pestis and Y. pseudotuberculosis branch but present in all other lineages. These data suggest that, like Salmonella enterica subsp. enterica serovar Typhimurium, Y. enterocolitica strains might be able to use naturally occurring carbon sources that are present in the inflamed gut but are not readily fermented, which gives these species a competitive advantage over the endogenous gut microbiota. Another important driver of bacterial disease, the chromosomal type III secretion system (T3SS), was also differentially present within Yersinia lineages. The T3SS, which facilitates the direct injection of effector proteins into host cells, seems to be in the process of being lost in the highly pathogenic species, and this loss coincides with the appearance of alternative T3SSs, either in the chromosome or in the virulence plasmid, $\mathrm{pYV}$.

Notably, the acquisition of $\mathrm{pYV}$ and the acquisition of the chromosomal attachment and invasion locus (ail) are the only two delimiters between the pathogenic strains and their closest environmental relatives. A phylogenetic analysis based on pYV segregates with the host lineages, despite the presence of fewer mutations than would be predicted from the genus tree. This unusual level of similarity between plasmids that are found at both extremes of the phylogenetic tree, coupled with the observation that closely related Y. enterocolitica biotypes have distinct pYV origins of replication, suggests that the different $Y$. enterocolitica lineages, as well as the Y. pseudotuberculosis and Y. pestis lineage, recently acquired distinct versions of $\mathrm{pYV}$.

Finally, the authors analysed the evolution of pathogenic lineages within Y. enterocolitica, and found that pathogenic biotypes had decreased metabolic capacity and increased functional gene loss when compared with non-virulent biotypes, in a similar pattern to that observed for Y. pseudotuberculosis and Y. pestis.

Taken together, these data show that pathogenic Yersinia species do not share a common ancestor but that they evolved independently and followed a parallel evolutionary pathway that includes the acquisition of the same virulence factors and progressive metabolic specialization. Cláudio Nunes-Alves

ORIGINAL RESEARCH PAPER Reuter, S. et al. Parallel independent evolution of pathogenicity within the genus Yersinia. Proc. Natl Acad. Sci. USA http://dx.doi.org/10.1073/pnas.1317161111 (2014) 